\title{
Spontaneous aortic arch thrombosis in a neonate
}

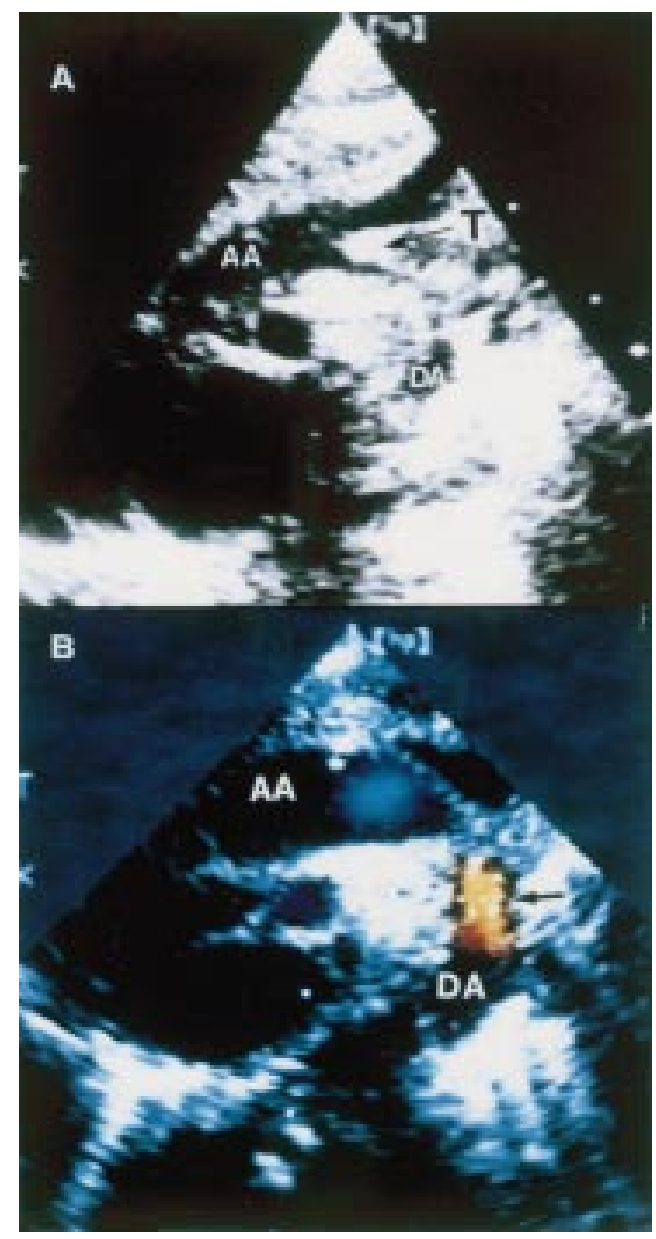

A male infant was born at 36 weeks' gestation weighing $2710 \mathrm{~g}$, after an uneventful pregnancy. Hypoxia was noted immediately following delivery $\left(\mathrm{O}_{2}\right.$ saturation $\left.85 \%\right)$, accompanied by tachypnoea, differential cyanosis (pale upper body and cyanotic lower body), and weak femoral pulses; blood pressure was $118 / 51 \mathrm{~mm} \mathrm{Hg}$ in the right arm and 48/ $17 \mathrm{~mm} \mathrm{Hg}$ in the right leg. Cross sectional echocardiogram (top left; high aortic arch view) shows an irregular thrombus $(T)$ at the aortic arch (arrow), as well as absence of blood flow in both carotid and left subclavian arteries.

Left ventricular contractility was reduced. Colour Doppler echocardiogram (bottom left) shows retrograde filling of the distal aortic arch via the patent ductus arteriosus (arrow). (AA, ascending aorta; DA, descending aorta.)

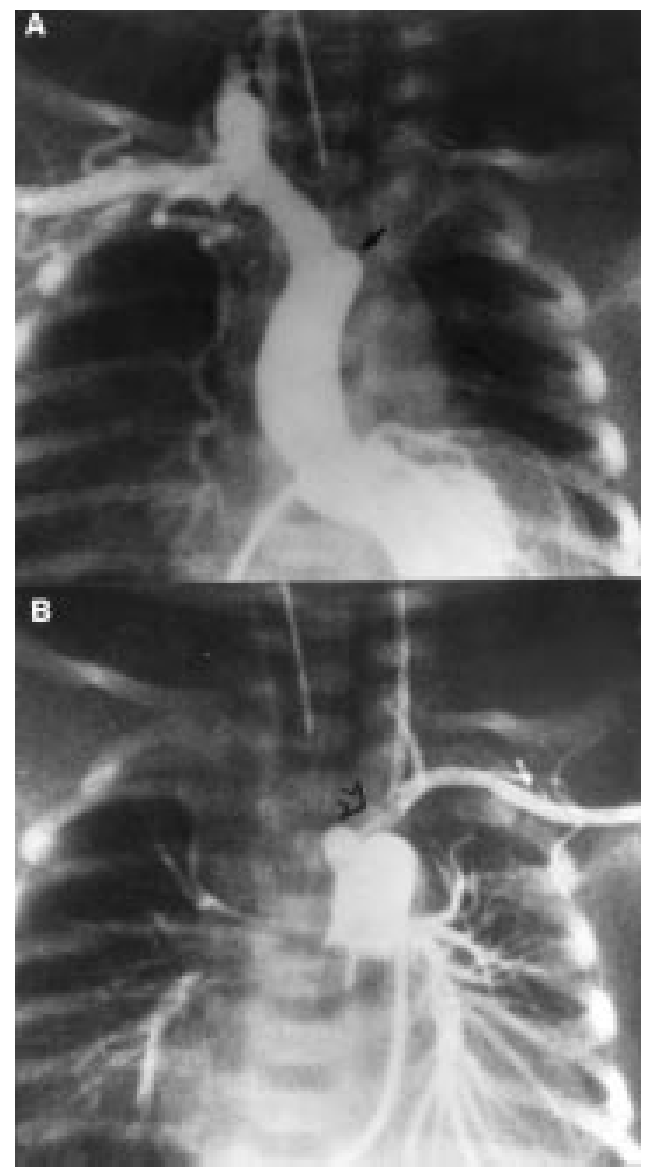

Left ventriculogram (top right) shows a dilated ascending aorta with complete interruption of the aortic arch just distal to the origin of the innominate artery by a thrombus (arrow head). There is a clear thrombus in the mid-right carotid artery (arrow). Balloon occlusion descending aortogram (bottom right) (via a patent ductus arteriosus) shows retrograde filling of the left subclavian artery and thrombus at its origin (arrow head), there is no filling of the left carotid artery.

Once the diagnosis of aortic thrombosis was confirmed, treatment with urokinase was started with almost complete resolution of the thrombus at 16 hours of age.

BENJAMIN ZEEVI MICHAEL BERANT 